\title{
Le système de riziculture intensive ou « SRI » à Madagascar
}

Entre légende urbaine et innovation rurale

\section{Georges Serpantié}

\section{OpenEdition}

\section{Journals}

Édition électronique

URL : http://journals.openedition.org/anthropodev/588

DOI : 10.4000/anthropodev.588

ISSN : 2553-1719

\section{Éditeur}

APAD - Association pour l'anthropologie du changement social et du développement

Édition imprimée

Date de publication : 1 décembre 2017

Pagination : 67-99

ISBN : 979-10-93476-05-6

ISSN : 2276-2019

\section{Référence électronique}

Georges Serpantié, «Le système de riziculture intensive ou «SRI » à Madagascar ». Anthropologie \& développement [En ligne], 46-47 | 2017, mis en ligne le 01 juin 2018, consulté le 30 avril 2019. URL : http://journals.openedition.org/anthropodev/588 ; DOI : 10.4000/anthropodev.588

La revue Anthropologie \& développement est mise à disposition selon les termes de la Licence Creative Commons Attribution 4.0 International. 


\title{
Le système de riziculture intensive ou « SRI » à Madagascar
}

\author{
Entre légende urbaine et innovation rurale
}

\author{
Georges Serpantié
}

Comment le système de riziculture intensive (SRI) - une invention malgache sans efficacité suffisante compte tenu de ses coûts selon l'agronomie expérimentale -, sans adoption importante, a-t-il pu néanmoins convaincre les nombreux acteurs, y compris une minorité de riziculteurs, qui l'ont promu, faire l'objet d'une politique nationale et, enfin, prospérer à une échelle internationale? En partant de l'hypothèse du "mythe sociotechnique", notre démarche allie agronomie et sociologie pour identifier les acteurs et les modes de construction des savoirs et des politiques agricoles, évaluer ce système de culture dans les conditions de la pratique, et comprendre les motivations du changement technique par l'exploitant. L'adoption politique du modèle SRI résulte d'une forte demande de renouvellement des messages techniques, dans un contexte de ruptures : changement politique à Madagascar et crise internationale $d u$ modèle "Révolution verte". Ce processus complexe associe des mécanismes cognitifs (I'illusion ou l'« autopromotion", la théorisation savante à l'échelle de la plante, le dogme) et des facteurs politiques (le "marketing", la légitimation d'un nouveau savoir, la mobilisation institutionnelle, le désir de distinction, et plus récemment la recherche d'éthique écologique). L'utilité pour la production ou l'agriculteur est intervenue marginalement, expliquant toutefois certains cas d'adoption authentique.

How did the system of rice intensification (SRI) - a Malagasy invention without sufficient efficacy given its costs according to experimental agronomy - and without significant adoption nonetheless convince the many actors - including a minority of rice farmers - who promoted it to become a national policy and, finally, thrive internationally? Drawing on the hypothesis of a "sociotechnical myth", our approach combines a sociological approach 
and an agronomic analysis to assess SRI at the farm level, to identify the mechanisms through which agricultural policies are shaped, and to understand farmers' motivation to change their practices. The political adoption process of SRI results from a need to renew technical messages in a context of major shifts: namely political change in Madagascar and the global crisis of the Green Revolution model. Cognitive mechanisms (illusion or "self-promotion", scholarly theorizing about the plant, dogma) and political factors (marketing, legitimization of new knowledge, institutional mobilization, the desire for distinction and, recently, the search for ecological ethics) are intertwined in these complex processes. The utility of SRI for production and for the farmer remained a marginal driver, however it explains some cases of genuine adoption.

\section{Introduction}

Sur la base d'un discours emphatique de technique révolutionnaire et scientifique, promettant l'autosuffisance alimentaire en 2000 (Laulanié, 1991, 1993, 1995 ; Vallois, 1996), un grand engouement médiatique a accompagné l'émergence du "système de riziculture intensive " (SRI) à Madagascar dans les années 1990 (Serpantié, 2013). Ce nouvel itinéraire technique était basé sur des repiquages de plants de 8 jours, à faible densité, dans peu d'eau, avec des sarclages mécaniques répétés et des assecs. Il était doublement alternatif : aux pratiques paysannes, et à la " riziculture améliorée » proposée par l'institution agronomique officielle depuis 1965. En tant qu'agronome d'un organisme de recherche contribuant aux débats du développement, nous voulons comprendre comment une idée technique sans efficacité suffisante au regard de ses coûts additionnels selon l'agronomie expérimentale (Razakamiaramanana, 1995 ; Dobermann, 2004 ; Tsujimoto et al., 2009), sans adoption massive sur le terrain (Moser et Barett, 2003), a pu néanmoins convaincre les nombreux acteurs, y compris une minorité de riziculteurs, qui l'ont 
promue, faire l'objet d'une politique nationale et prospérer à l'échelle internationale ${ }^{1}$.

La discordance entre l'engouement politique pour le SRI et sa faible adoption pratique est un indice de ce que les critical political ecologists appellent des « mythes " (Forsyth, 1996). II s'agit de raccourcis logiques, fallacieux à certains égards, mais institutionnalisés, devenant des orthodoxies de pensée. Le phénomène SRI pourrait même avoir un rapport avec les légendes urbaines, ces histoires modernes proches de la rumeur, analysées par le sociologue Edgar Morin (1969). Comprendre le mythe, s'il s'agit de cela, nécessite une étude des rapports entre pratiques, politiques et savoirs, objet de la sociologie des sciences, à laquelle nous emprunterons des outils d'analyse.

Le savoir est un capital individuel de compétences, d'expériences et d'enseignements retenus. Mais un groupe partage aussi un savoir commun. Le savoir total d'une société est hétérogène (traditionnel, religieux, scientifique...) et traversé d'oppositions (profane/savant, autodidacte/académique, universel/local et même autorisé/non reconnu). En effet un acteur social construit son savoir en en rejetant d'autres (Verrier, 1999). La science est un mode particulier de construction d'un savoir universel, mais en évolution. Pour Callon et al. (2001), l'histoire des sciences depuis "l'esprit de curiosité » des élites montre un processus de confinement dans des laboratoires d'État. Les chercheurs sont ensuite partis sur le terrain se confronter à la complexité. La science officielle est aujourd'hui contestée par une société en perte de confiance. Elle lui préfère parfois une science populaire, construite par des autodidactes, qui font appel aux savoirs profanes. L'agronomie est aussi confrontée à cette contestation (Sumberg et al., 2012).

Dans ce contexte de tensions cognitives, une nouvelle sorte de forum apparaît : les forums hybrides (Callon et al., 2001). On y examine faits et solutions de différents points de vue, profanes ou savants. Cette nouvelle

\footnotetext{
${ }^{1}$ La base de données de I'université Cornell sur le SRI contient un millier de références, montrant l'engouement scientifique pour le sujet (https://www.zotero.org/groups/system _of_rice_intensification_sri_research_network/items/).
} 
science démocratique permet de discuter d' "options oubliées" et de mener vers des savoirs co-construits. Les chercheurs résistent aussi à leur marginalisation en s'engageant et en se structurant en communautés épistémiques. II s'agit de groupes d'experts reliés par une même pensée, qui influencent la création de nouvelles politiques, leur diffusion, ou le maintien de certaines idées à l'agenda politique en rejoignant des coalitions de plaidoyer (Adler et Haas, 1992).

Ce premier cadre permet donc de poser diverses questions sur les processus d'élaboration du SRI en tant que nouveau savoir à succès : à quel registre de savoirs appartiennent le SRI et ses discours de justification ? La genèse du SRI relève-t-elle d'un tel processus de contestation du savoir scientifique ou de ses préconisations ? Le SRI peut-il être considéré comme une de ces "options oubliées" examinées dans le cadre d'un forum hybride ? Le maintien du SRI à l'agenda international est-il le fruit d'une coalition d'acteurs notamment scientifiques? Pour répondre à ces questions nous nous sommes attaché à mener une revue bibliographique historique exhaustive ${ }^{2}$ (période 1983-1995) et avons recueilli une dizaine de témoignages (chercheurs, responsables de projets, leaders agricoles, par entretien ou courrier) sur la genèse du SRI à Madagascar en vue d'analyser les rôles des acteurs institutionnels, et particulièrement des acteurs de la sphère cognitive.

Une pratique agricole comme le SRI est toutefois inscrite à la fois dans la société et le monde biophysique. Son étude nécessite donc un cadre d'analyse interdisciplinaire. L'agronomie a développé dans les années 1970 des concepts d'analyse de la parcelle cultivée, système piloté par l'homme en milieu changeant, ainsi que de l'exploitation agricole, unité de prise de décision (Sebillote, 1974 et 2006). L'agronomie des pratiques s'est penchée sur les techniques adoptées par les exploitants agricoles et sur leurs différentes dimensions, notamment économiques (Milleville, 1987). C'est

${ }^{2}$ Tandis que la base de données sur le SRI de l'université Cornell contient un millier de références, paradoxalement, Madagascar, berceau du SRI, n'en compte que 13, et une seule sur la période 1983-1995 pourtant période d'émergence, ce qui nous a obligé à une recherche d'archives. Nous avons collecté une quinzaine de textes permettant de documenter cette période. 
ce cadre que nous avons mobilisé ici pour évaluer le SRI « mis en pratique ", sous l'hypothèse que les résultats du SRI (production, performances environnementales, coûts, adoption) sont encore mal connus à Madagascar, expliquant une grande partie du mythe présumé. Nous nous appuyons sur la bibliographie disponible complétée par une enquête agronomique menée par nous-même entre 2006 et 2008 dans la région de Fianarantsoa (Serpantié et Rakotondramanana, 2013).

Notre analyse comprend quatre parties, selon un plan chronologique : le contexte, la genèse du discours, les évaluations, et la mise en politique.

Riziculture et contexte politique malgache : des ruptures

Le riz est aux sociétés malgaches des Hautes Terres une norme alimentaire, la base des échanges sociaux, et un symbole majeur. Mais les pratiques rizicoles diffèrent entre régions, au sein de chaque localité et même au sein de l'exploitation agricole (Le Bourdiec, 1974). Cette dernière conduit plusieurs types de rizières, sur plusieurs "saisons", avec plusieurs variétés, et plusieurs gestions de la fertilité et de l'eau (Radanielina et al., 2014). Il existe toutefois des préférences et normes régionales en matière de variétés, modalités de préparation du sol et de repiquage ${ }^{3}$ (fig. 1).

Dans la région de Fianarantsoa, les rizières paysannes produisaient, en 1965 , en moyenne $2,1 \mathrm{t} /$ ha $(+/-0,3)$ de paddy, sur une gamme de 0 à $7 \mathrm{t} / \mathrm{ha}$ (INSRE, 1965). Cette faible moyenne ancienne a permis aux agents de développement rural de décrire cette agriculture locale par une notion simplificatrice : le fomba taloha, c'est-à-dire " modèle antique " ou encore "SRT» (système de riziculture traditionnelle) "produisant $2 \mathrm{t} /$ ha en moyenne " (Gillain, 1992 ; Laulanié, 1993 ; Stoop et al., 2002 ; FOFIFAASARECA, 2013). Ces qualificatifs, cette singularisation et ce chiffre rond et invariable malgré 50 ans d'évolution servent encore aujourd'hui de référence aux promoteurs d'une intensification agricole.

\footnotetext{
${ }^{3}$ Pour les rizières de bas-fonds vers Fianarantsoa, il en va ainsi de la pratique vivace du piétinage animal, des variétés de riz rouge, de certains traits du mode de plantation (en foule dense, plusieurs plants par poquet, cf. fig. 1). L'inondation est quasi continue et les désherbages sont manuels.
} 


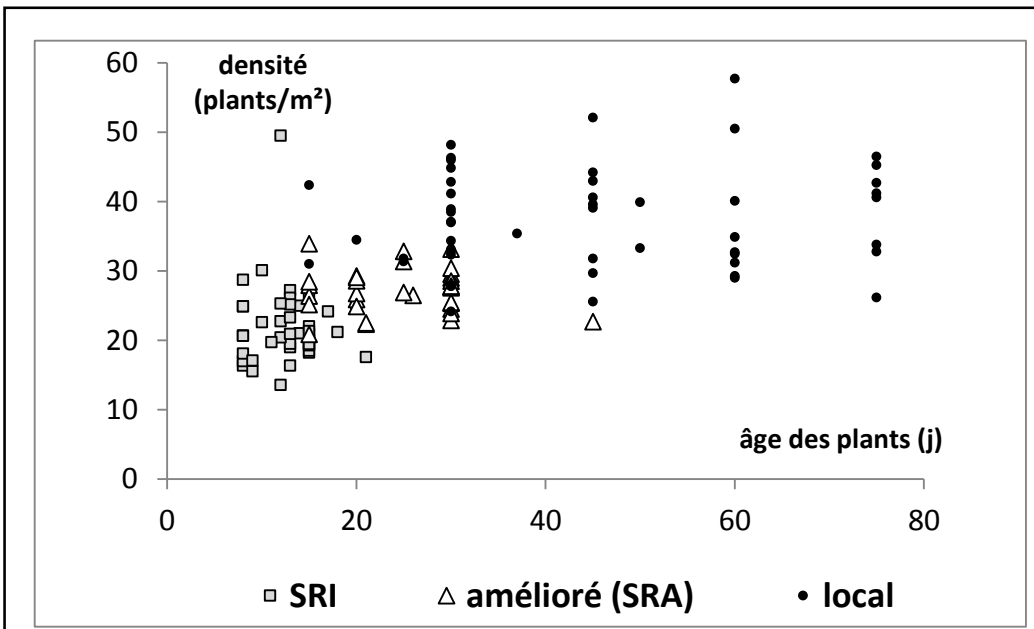

Figure 1. Modes de repiquage des rizières de bas-fonds observés vers Fianarantsoa (données Serpantié et Rakotondramanana, 2013).

La première norme technique diffusée fut une forme de riziculture légèrement mécanisée appelée "méthode améliorée de riziculture » (MAR), théorisée par la recherche agronomique franco-malgache (notamment I'IRAM ${ }^{4}$ ) et introduite par les services agricoles et projets d'État des années 1960 (Dufournet et Roche, 1967; Gillain, 1992). Requalifiée aujourd'hui système de riziculture améliorée (SRA), cette méthode est basée sur un repiquage en ligne (donc une moindre densité), certains intrants et matériels (variétés nouvelles, traitements des semences, fertilisation équilibrée, sarcleuse mécanique), et préconisations (plants de moins de 30 jours) (fig. 1). L'intérêt économique mena à une adoption de ce modèle dans les régions de grandes exploitations comme I'Alaotra. La région de Fianarantsoa a été moins enthousiaste, seulement $13 \%$ de la surface rizicole était repiquée en ligne en 2001 (MAE-UPDR, 2002). Les exploitations y sont restées de petites structures, peu monétarisées. Les variétés locales y restent majoritaires (Radanielina et al., 2014).

${ }^{4}$ Institut de recherche agricole de Madagascar. 
Le recours aux intrants onéreux augmente seulement lorsqu'il existe un soutien organisé de crédits ou subventions.

Les années 1985-1995 marquent une période de libéralisation à Madagascar après deux régimes dirigistes caractérisés par des crises financières et des relations diplomatiques peu diversifiées (Randriamamonjy, 2009). Après la dévaluation de 1986-1988, les plans d'ajustement structurel de 1989-1993 mettent l'État sous tutelle internationale. L'État est invité à se désengager de ses activités de développement au profit des ONG et à se recentrer sur ses rôles régaliens. La crise insurrectionnelle pour la démocratie de $1991^{5}$ a initié la transition démocratique et libérale de 1991-1993, marquée encore par des tensions mais aussi par le développement de médias pluralistes et le lancement de plans soutenus par la Banque mondiale (BM) et d'autres bailleurs bilatéraux (USAID, AFD, etc.) dans les domaines agricoles (Programme national de vulgarisation agricole ou PNVA) et environnementaux (Plan national d'actions environnementales ou PNAE).

À cette rupture politique s'ajoutait une économie agricole en crise. Le contexte de cherté des intrants et de faible prix du riz remettait en cause la rentabilité du modèle rizicole MAR et relativisait l'intérêt des activités de I'IRRI $^{6}$, champion de la "Révolution verte " qui menait des recherches variétales rizicoles avec l'organisme agronomique malgache, le FOFIFA. À l'absence de réponses aux préoccupations du secteur agricole, s'ajoutait l'influence d'un discours environnementaliste international, réintroduit à Madagascar en 1984 par l'ONG WWF, appelant à réduire la pression sur les milieux naturels, particulièrement dégradés au cours du régime antérieur. C'est dans ce contexte de ruptures et de crises multiples que l'État adopte une nouvelle norme, le SRI, en 1995.

\footnotetext{
${ }^{5}$ Le Conseil des églises chrétiennes de Madagascar (FFKM) a joué un rôle structurant dans le mouvement d'opposition "Forces vives" en convoquant le premier congrès dit «Concertation nationale des Forces vives » en août 1990 (Randriamamonjy, 2009).

${ }^{6}$ International Rice Research Institute.
} 


\section{Une idée germe puis circule}

Ce contexte très demandeur d'alternatives fournissait un programme aux agronomes: rechercher des systèmes de culture intensifs moins consommateurs d'intrants importés. Des recherches franco-malgaches sont alors lancées sur le fonctionnement des bas-fonds rizicoles, l'amélioration des petits périmètres irrigués, la création variétale dans d'autres directions (riz pluvial adapté à l'altitude). Pour l'IRRI, appuyé par les USA, des approches intégrées (farming systems research) s'ajoutent à l'amélioration des plantes. La recherche agronomique se mène aussi dans des cadres non gouvernementaux. C'est dans ces derniers que le SRI prend sa source.

\section{Conception du SRI}

Henri de Laulanié (1920-1995), inventeur du SRI, était un prêtre jésuite, ingénieur agronome. Arrivé à Madagascar en 1961, il enseignait depuis 1972 l'agriculture au Centre de formation artisanal, agricole et ménager (CEFAAM) de Mahitsy, près de la capitale, ainsi qu'en province.

À la fin de 1983, ses séminaristes du Centre de formation rurale St Jean de Manantenasoa (près d'Antsirabe) observaient par hasard la capacité de plants âgés de 15 jours, repiqués dans très peu d'eau et à faible densité, de taller ( $c^{\prime}$ est-à-dire ramifier) généreusement. Laulanié répéta cette expérience chaque année, avec des plants plus jeunes (GSRI, 2012). L'IREDEC, une ONG catholique d'Antsirabe, l'aida à disséminer sa " méthode des plants de 8 jours" (ketsa zatoandro), à partir de 1987, dans les communautés rurales catholiques près d'Antsirabe (Mouroux, 2008). En 1990 fut fondée I'ONG Association Tefy Saina (ATS), "forger l'esprit ", pour diffuser largement la nouvelle méthode bien qu'aucun écrit n'ait été publié.

Un feuilleton sort dans un hebdomadaire catholique en mai et juin 1991 pour présenter la base théorique de " la riziculture scientifique basée sur le modèle de tallage de Katayama " (Laulanié, 1991). L'itinéraire technique est décrit en détail en 1993 sous le nom de "système de riziculture intensive " ou "SRI » (Laulanié, 1993). De très jeunes plants (stade 2 feuilles) obtenus en pépinière sèche sont soigneusement repiqués dans une boue collante, un par un, à faible densité (env. $16 / \mathrm{m}^{2}$ ), en lignes 
croisées. En phase végétative, des périodes d'inondation légère alternent régulièrement avec des assecs réalisés après sarclage à la houe mécanique. Le SRI prendra en 1995 le nom de voly vary maro anaka (riziculture à nombreuse progéniture) lors de son adoption nationale.

Ces principes culturaux n'étaient pas tous nouveaux. Mais l'apport innovant a été d'en faire un système cohérent, comme l'ont montré les tests agronomiques de variation des composantes ${ }^{7}$ les unes par rapport aux autres (Razakamiaramanana, 1995). C'est aussi un système technique doublement alternatif. Le SRI se situe au bout de la trajectoire de changement du mode de plantation initiée par la "riziculture améliorée ", exploitant la dernière zone encore inoccupée dans l'espace des caractéristiques techniques du repiquage (fig. 1). Ce modèle n'avait pas de domaine de recommandation autre que "les rizières à bonne maîtrise d'eau ». Normatif sur les phases plantation et entretien, il était en revanche ouvert sur tout le reste du système de culture: modes de préparation du sol, entretien de la fertilité, lutte contre les ravageurs, successions et choix variétal. Les promoteurs organisaient cependant des concours de rendement, d'abord officieux, ce qui était une incitation à poursuivre cet objectif par une intensification complète du système de culture.

\section{La rhétorique $S R I$}

L'argumentaire de promotion promet un gain substantiel de rendement sans intrants supplémentaires. Il devient de plus en plus emphatique: "Les rendements sont ainsi passés de 2 tonnes de paddy à l'hectare à 8 voire 12 tonnes avec des variétés locales" (Laulanié, 1993: 110). "Doublement ou quadruplement des rendements ? " (Vallois, $1996: 21$ ). Ce langage multiplicatif est repris sans réserve par des médias nationaux comme internationaux (Ratsimbarison et Williams, 1993). Dès l'adoption nationale en 1995 à l'occasion de "l'Année nationale du riz ", le concours de rendement organisé par l'ONG ATS devient officiel et placé sous l'égide du ministère de l'Agriculture. Les prix sont décernés lors de la Fête

${ }^{7}$ C'est-à-dire la densité de plants, le nombre de plants par touffe, l'âge des plants. 
nationale. ATS communique alors régulièrement des records ahurissants : après 15 t/ha en 1993, on annonce 24 t/ha en 2003 (ATS, 2007).

Les promoteurs initiaux et leurs successeurs proposent une explication scientifique à cette multiplication des rendements : le fait que le repiquage à faible densité de plants juvéniles augmente la capacité de tallage du pied (Laulanié, 1991 ; Vallois, 1996; Uphoff, 1999; Stoop et al., 2002). L'iconographie des fiches techniques confirme que l'argumentaire concerne surtout "l'échelle " du pied de riz avec un objectif de prolificité. Dans la figure $2 \mathrm{a}$, le grain de riz levé au stade " 2 feuilles " est assimilé à une jeune femme féconde (en malgache, l'épiaison se dit "accouchement "), et le SRI est symbolisé par un pied de riz solitaire chargé de panicules (fig. 2b).

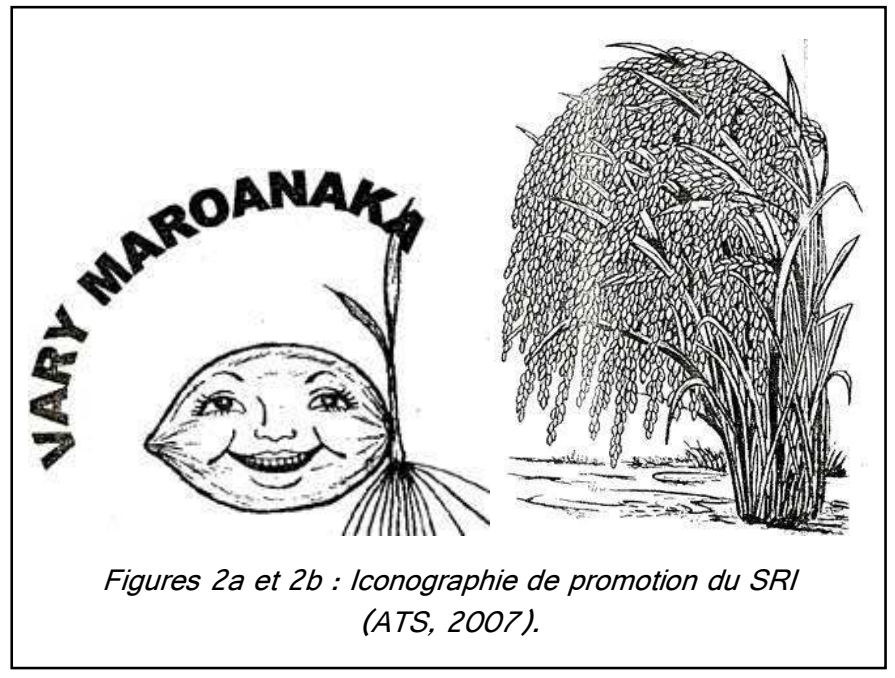

Cette argumentation scientifique reste évidemment réductrice pour une technique agricole visant un accroissement de productivité, qui implique d'autres échelles que le pied (peuplement, parcelle, exploitation).

${ }^{8}$ Un autre argument porte sur l'oxygénation de la zone racinaire. 
L'inventeur ajoute des arguments renvoyant à des valeurs sociales. Outre l'exaltation du savoir et du travail, la pratique des paysans est fustigée, décrite comme "barbare " (Laulanié, 2003 : 74). Le traitement brutal infligé aux jeunes plants (arrachage, nettoyage) est jugé traumatisant et la promiscuité des plants impliquerait une compétition épuisante, alors qu'au sein d'un pied les talles " s'entraident ". Les images anthropomorphiques (fig. 2) et les formes de personnification du riz abondent: "Le dernier mot appartiendra au riz lui-même. À nous de savoir l'écouter » (Laulanié, 1991: 5). En recourant au modèle de Katayama et à la précision de la prescription, l'inventeur juge sa proposition "scientifique " (ibid.) : "Fini la riziculture de l'à-peu-près » (Laulanié, 1993 : 113).

Cette rhétorique alliant promesses de gains prodigieux et explications à la fois scientifiques, imagées, et porteuses de valeurs, reprise presque mot pour mot par les héritiers de l'inventeur, aura de multiples effets sur les autres acteurs du développement : séduire les uns, exaspérer les autres, attirer les médias et des courtiers prêts à diffuser le message. L'approche compétitive poussera aussi les premiers adeptes à rechercher un record de rendement en jouant sur la partie libre de l'itinéraire technique.

Modérée par certaines ONG qui n'évoquent plus que des gains moyens de rendement de $47 \%$ (Africare, 2010), ce qui est encore considérable, l'emphase ressurgit aujourd'hui dans les revues de vulgarisation agroécologique consacrées au SRI ${ }^{9}$. Ces annonces à la logique approximative, comparant des maximums et des moyennes, des données actuelles et anciennes, des données vérifiées et non vérifiables, rivalisaient avec les annonces des tenants de la Révolution verte qui promettaient depuis les années 1970 de hauts rendements sur la base d'un changement variétal ${ }^{10}$.

\footnotetext{
${ }^{9}$ " II permet une productivité de $20 \mathrm{t} /$ ha dans les conditions optimales au moment où les systèmes traditionnels n'en permettent pas plus de $2 \mathrm{t} /$ ha à Madagascar " (Agridape, $2013: 4)$.

${ }^{10}$ En omettant de dire que les conditions économiques (rapports des coûts d'intrants aux prix des produits) ne permettraient le plus souvent l'expression de ces hauts rendements que sur les plus grands domaines.
} 


\section{L'évaluation du SRI}

Le SRI a été promu dans la région d'Antsirabe dès 1987, autorisé à la diffusion nationale en 1992 (Laulanié, 1993) puis adopté par les autorités nationales dès 1995 (Vallois, 1996). Pendant cette période, aucune évaluation agronomique ou économique, ni interne, ni indépendante, n’a eu lieu, laissant libre cours au discours de promotion.

\section{Pas d'évaluations agronomiques ni économiques avant l'adoption nationale}

Le discours théorique de la première publication dans une revue confessionnelle (Laulanié, 1991) donnait encore peu de prise à une lecture agronomique. L'itinéraire technique n'était abordé qu'en termes de " principes ». II fut précisé en 1993 mais les rendements avancés étaient des "records" donc les maxima d'un grand nombre de parcelles d'apprentissage et de concours, sans comparaison avec les records d'autres pratiques. Aucun résultat d'essais comparatifs " toutes choses égales par ailleurs " n'était jamais apporté, et encore moins d'évaluations économiques. Des explications de la technique, une théorisation de type écophysiologique et l'absence de résultats expérimentaux comparatifs caractérisaient les deux textes de présentation du SRI à destination du monde savant (Laulanié, 1993 et 1995).

Vallois (1996), avocat du SRI auprès des ONG françaises et malgaches, écrit que l'inventeur ne voulait pas entendre parler d'essais contradictoires. Il admet aussi une rhétorique excessive, un " pieux mensonge " comme stratégie de communication à visée populaire afin de lancer une " agriculture rationnelle » (ibid. : 22).

L'absence de réactivité précoce de l'agronomie officielle, que l'on pourrait imputer à un manque de curiosité, est aussi le résultat d'une stratégie d'évitement de la part des promoteurs. Bien que très proches, à Mahitsy (où FOFIFA et IRRI menaient leurs essais variétaux) comme à Antsirabe (où travaillait le CIRAD), les chercheurs et l'inventeur appartenaient à des "sphères" agronomiques séparées. Ainsi, en décembre 1991, le séminaire d'Antananarivo "Bas-fonds et riziculture » marquant la fin d'un programme de recherche franco-malgache sur les 
bas-fonds ${ }^{11}$ a produit un document de référence (Raunet, 1993) : il ne fait pas allusion au SRI. Ni Laulanié, ni l'IREDEC, ni l'ATS, n'ont été comptés parmi les participants officiels, mais l'inventeur a assisté au moins aux sessions sur l'écophysiologie du riz (Laulanié, 1993). Il a aussi, par contre, pu communiquer sur le SRI auprès des ONG françaises présentes (Lavigne Delville, communication personnelle). Les chercheurs FOFIFA assistaient néanmoins aux séminaires annuels organisés par Laulanié à Mahitsy dès mi- $1989^{12}$, où la théorie professée ne souffrait aucune contradiction, et où ils étaient noyés parmi les fonctionnaires de plusieurs ministères, des personnels diplomatiques, ceux des organisations internationales, et même des services agricoles de l'armée (chercheurs FOFIFA, communications personnelles).

Pour les chercheurs officiels, le sujet était déjà politiquement sensible. Ainsi D. Rollin (CIRAD) travaillant à Antsirabe pour le projet "Petits Périmètres irrigués " fut finalement invité par l'IREDEC à une visite de parcelles SRI en mars et avril 1992, signe que cette ONG cherchait un partenariat scientifique. Dans son rapport nuancé, il reconnaissait aux principes du SRI un pouvoir d'accroissement du rendement et voyait des agriculteurs convaincus. Mais il soulignait aussi des limites, ainsi que des confusions dans les justifications théoriques. II conseillait la réalisation d'un programme d'essais pour préciser le domaine de recommandation (Rollin, 1992). Malgré ce rapport intéressé et constructif, il fut convoqué quelques mois plus tard pour un débat contradictoire avec Laulanié dans le bureau du ministre de l'Agriculture. Ce qui montre la grande proximité de I'inventeur avec les autorités dès 1992, avant même que sa proposition ne soit précisée et publiée, et une stratégie de débat arbitré par le politique.

Le programme pilote de développement agricole préparant le PNVA recherchait des thèmes à vulgariser. Un programme de tests de terrain du SRI en 1993-1994 fut lancé où le SNVA (Service national de vulgarisation agricole) collaborait directement avec ATS, mais sans appui scientifique. Les résultats étaient à nouveau emphatiques et sans explications métho-

\footnotetext{
${ }^{11}$ Le programme ATP-PIREN CNRS-FOFIFA-ORSTOM-CIRAD-LRI-Univ. Montpellier et Avignon.

${ }^{12}$ Le SRI n'y sera promu qu'à partir de mi-1990.
} 
dologiques $(B M, 1995)^{13}$. Mais l'inventeur visait plus haut : il en appelait à I'IRRI pour valider le SRI. Dans sa réponse en mars 1993, le directeur général de l'IRRI aux Philippines lui a témoigné d'un intérêt poli, n'y voyant rien de neuf (Vallois, 1996 : 87). Le FOFIFA-IRRI lancera finalement un essai en partenariat avec IREDEC fin 1994. Les chercheurs, confrontés au SRI, n'avaient donc pas encore de données opposables au moment de l'adoption nationale en 1995. Mais les promoteurs obtenaient in extremis une légitimation académique ${ }^{14}$.

\section{Premières évaluations agronomiques avortées et censures}

L'expérimentation FOFIFA-IRRI livra ses conclusions en décembre 1995, après le décès de Laulanié. Outre des essais d'optimisation du SRI (par réglage de chaque "composante" l'une par rapport aux autres), Razakamiaramanana (1995) comparait 4 systèmes sur des parcelles de $50 \mathrm{~m}^{2}$ sans répétition : le système dit traditionnel (variété locale repiquée en foule), le système dit amélioré (variété améliorée repiquée en lignes), le $\mathrm{SRI}$, et enfin un système dit amélioré intensif. Le système traditionnel donnait $4,4 \mathrm{t} / \mathrm{ha}$, le SRI 4,6 t/ha (soit $+5 \%$ ), le système amélioré $4,8 \mathrm{t} / \mathrm{ha}$ $(+9 \%)$ et le système amélioré intensif $5,4 \mathrm{t} / \mathrm{ha}(+23 \%)$, ce dernier étant " non rentable ». Tout en rappelant que la vulgarisation du SRI était à ce jour déjà appuyée par l'État (ibid. : 10), l'article jugeait négatif l'intérêt du SRI, dont le surcoût en travail n'est " pas compensé par l'économie de semence, d'eau, de temps et le surplus de rendement » (ibid. : 12).

Si cette première étude comparative à l'échelle " parcelle ", bouclée en un temps record, arrivait trop tard pour éclairer les décideurs, elle confirmait le caractère abusif du discours de promotion en montrant que l'ordre de grandeur des différences entre systèmes de culture restait

\footnotetext{
${ }^{13}$ Le SRI (maîtrise d'eau et plants de 8 jours) gagne de $69 \%$ à $258 \%$ de rendement par rapport au témoin (BM, $1995: 22$ ). Mais le simple repiquage de plants plus jeunes sur rizières sans maîtrise d'eau donne aussi de +43 à $251 \%$. Tous les autres thèmes testés par le SNVA font état de gains du même ordre de grandeur.

${ }^{14}$ Une communication de nature théorique à l'Académie malgache (Laulanié, 1995) apportait la consécration tant attendue en février 1995, peu avant "l'adoption officielle " et le décès de l'inventeur.
} 
faible. En revanche, elle restait encore insuffisante sur le plan statistique, n'enseignait rien sur les éventuelles variations d'efficacité du SRI en fonction des situations, et n'expliquait pas pourquoi on signalait des records. Critiqué au ministère pour cet article qui tombait peu à propos, l'auteur de cette première étude a abandonné ce thème de recherche qu'il aurait fallu mener sur plusieurs années et plusieurs sites ${ }^{15}$.

Le SRI étant devenu une nouvelle norme d'État vulgarisée dans tous les projets rizicoles, le FOFIFA et l'université agronomique ESSA initièrent dès 1998 avec l'appui du CIIFAD ${ }^{16}$ (Université Cornell) des recherches d'optimisation du SRI (Andrianaivo, 2003), le prenant désormais comme cadre de travail concurrent de la MAR renommée pour l'occasion « SRA ».

\section{Premières évaluations socio-économiques}

La première analyse comparée à l'échelle de l'exploitation viendra plus tard, de socio-économistes d'un autre programme de Cornell (ILO) travaillant sur les terrains de vulgarisation du SRI autour du parc national de Ranomanafa. Constatant la désadoption du SRI après apprentissage, ils pointaient surtout le problème des coûts en travail élevés du SRI (Moser et Barrett, 2003). D'autres auteurs relevaient des facteurs socioculturels contrariants comme l'égalitarisme ou l'inertie paysanne (Gannon et Sandron, 2003 ; Bockel, 2005). Ils entérinaient en revanche le discours sur le rendement, faute de pouvoir le questionner par eux-mêmes, sauf par des sondages dans les parcelles, mais sans appliquer le principe «toutes choses égales par ailleurs " ni vérifier la représentativité des témoins. Ainsi ont-ils renforcé les promoteurs, doublement : en reconnaissant l'effet sur le rendement et en justifiant la valeur " travail » attribuée au SRI.

\footnotetext{
${ }^{15}$ À partir des données de l'essai FOFIFA-IRRI de 1994-1995, I'homologue américain de l'IRRI, M. Gaudreau, a même produit une forme de repentir (Razakamiaramanana et Gaudreau, 2000). Ce poster à la rencontre annuelle de la Société américaine d'agronomie partait du postulat de " doublement de rendement » (contredisant donc Razakamiaramanana, 1995) et vulgarisait l'hypothèse de « synergie des composantes » de Uphoff (1999).

${ }^{16}$ Cornell International Institute for Food, Agriculture and Development.
} 


\section{Évaluation du SRI mis en pratique}

Comprendre le fonctionnement comparé et les coûts du SRI à l'échelle de la parcelle requiert une approche agronomique, soit par des essais multilocaux nécessitant de gros moyens, soit en profitant de la mise en pratique embryonnaire observée sur le terrain.

Un peuplement SRI est d'abord très reconnaissable, ce qui est indispensable aux mesures incitatives. II se distingue par des pieds de riz particuliers, à savoir de grosses touffes chargées de tiges fertiles, vertes, quand les rizières voisines montrent un peuplement dense de pieds de quelques tiges, parfois jaunâtres (fig. 3). Ce pied de riz géant et le " nombre d'épis par pied " que l'on compte devant les caméras sont très séduisants pour les « non-paysans ».

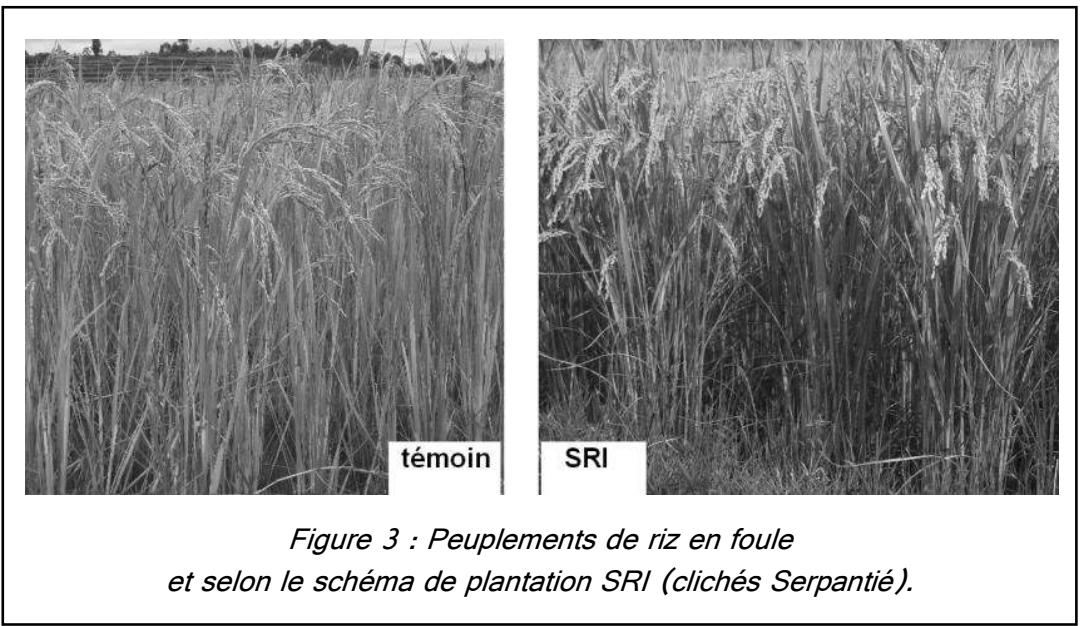

Nos études dans la région de Fianarantsoa (Serpantié et Toillier, 2007) et sur une centaine de parcelles paysannes (Serpantié et Rakotondramanana, 2013) ont donné les résultats suivants :

- Suite à la diminution des superficies par habitant (de 1030 ares/hab. dans les années 1960 à 5-10 ares/hab. en 2004), les pratiques locales actuelles n'ont jamais été aussi intensives et productives avec un rendement moyen de $4 \mathrm{t} /$ hectare et une charge de travail moyenne de 225 journées de travail/hectare. 
- Les agriculteurs adoptants respectent en grande partie les itinéraires techniques recommandés en SRI et SRA, mais affectent leurs meilleurs sols et de fortes doses de fertilisants au SRI. Le coût en travail augmente de $60 \%$ pour le SRI et s'y ajoutent des coûts en monnaie (opérations salariées - plantation, sarclages mécaniques - et engrais minéral). Le SRI est donc bien intensif, coûteux en travail et en monnaie mais permet toutefois de réduire la dose de semences.

- Les rendements bruts du témoin local et du SRA ne sont pas significativement différents. Le rendement brut moyen du SRI augmente de $24 \%$, significativement par rapport au témoin, mais la productivité brute du travail ne change pas significativement.

- À dose de fumure égale et en regroupant les situations de même nature, les conclusions sont plus précises sur les effets nets :

- $\quad$ En cas de sécheresse, le SRI donne moins de panicules $/ \mathrm{m}^{2}$ que le non-SRI, et le rendement SRI baisse de $7 \%$. La pratique paysanne de fortes densités de repiquage est efficace pour limiter la perte de panicules.

- Sans sécheresse, sur sols ordinaires, il y a confusion entre effet fumier et effet SRI. Le SRI n'a pas d'avantage additionnel net sur le non-SRI.

- La situation " sans sécheresse, sols fertiles " (un tiers des cas) procure seule un avantage net au SRI de $+16 \%$. Le $\mathrm{SRI}$ réduit alors certains facteurs limitants. Comme ce sont déjà les meilleurs rendements du témoin, ce gain supplémentaire produit un record ( $8 \mathrm{t} / \mathrm{ha})$, supérieur au maximum du témoin ( $7 \mathrm{t} / \mathrm{ha})$.

Dans la région étudiée, le gain net moyen pondéré de rendement toutes choses égales du SRI n'est finalement que de $+5 \%$, et la productivité du travail toutes choses égales du SRI chute drastiquement de $50 \%$. Les records de rendement sont donc trompeurs: ils masquent I'inefficacité moyenne du SRI à dépasser les autres pratiques, toutes choses égales, sans parler du coût élevé de mise en œuvre et des risques de pertes en cas de sécheresse au départ, ce qui est de plus en plus fréquent à Fianarantsoa. 
Les enquêtes soulignent aussi que les taux d'adoption sont plus élevés près des routes et marchés (Serpantié et Rakotondramanana, 2013). II s'agit souvent de paysans aisés qui possèdent des moyens adéquats (Moser et Barett, 2003) $^{17}$ et des rizières idéales. Mais la principale cause d'adoption dans la région d'étude n'est pas l'intérêt pour la technique en elle-même, mais pour ce qui l'accompagne. Ainsi le SRI est très bien adopté à Ambatovaky, village "vitrine" du SRI sur la route du parc de Ranomafana. II y a bénéficié de la constance d'un " champion local » du SRI depuis 1993 (un néorural devenu président de la Coopérative de I'Union des Groupements Koloharena). Ce leader a su attirer depuis 1995 des incitations continues à pratiquer le SRI (formations, financements, engrais à crédit, matériel) ${ }^{18}$. En revanche, dans les villages lambara et Torotosy où les incitations ont décru après la période de formation ATSCIIFAD 1995-1998, on a assisté à une désadoption. Les paysans cherchent donc surtout à profiter de la rente économique et sociale que l'engouement institutionnel a générée.

Les néoruraux (retraités, instituteurs, jeunes de retour au village) ont un discours de distinction. Non seulement la pratique du SRI les distingue des paysans, mais ils accèdent grâce à elle à des mesures d'accompagnement et à des responsabilités (diplômes d'agriculteur " professionnel ", statut de paysan-formateur, financements, etc.). Enfin, des femmes en sont adeptes: soit pour les mêmes raisons, soit parce qu'elles sont employées comme repiqueuses SRI à un tarif plus intéressant, soit parce qu'elles apprécient la pépinière courte facile à conduire seules, ainsi que l'économie de semences.

\footnotetext{
${ }^{17}$ Bétail donc fumier, bonne maîtrise d'eau, sols minéraux portants et plus fertiles, cultures de contre-saison fumées, moyens de payer une main-d'œuvre supplémentaire qualifiée.

${ }^{18}$ Le dernier projet AROPA-FIDA-AFDI en 2013, par exemple, fournissait de l'engrais chimique subventionné à condition de cultiver au moins 5 ares en SRI, ce qui a accru l'adoption apparente jusqu'à $70 \%$ en 2013.
} 
Une innovation d'abord institutionnelle

L'analyse de l'adoption institutionnelle du SRI montre des processus semblables à toutes les échelles, depuis la région d'origine, l'échelle nationale, puis internationale; chaque extension d'échelle relégitimant les actions à l'échelle inférieure et apportant des moyens nouveaux pour le fonctionnement du réseau et les incitations.

\section{La segmentation du secteur du développement de la riziculture malgache}

Cette proposition technique, avec ses méthodes de diffusion, s'est développée d'abord au sein d'un milieu particulier: les ONG de l'église catholique, à la fois puissante à Madagascar et en marge des institutions scientifiques et officielles, ce qui a permis la maturation de l'innovation en vase clos et un renforcement des convictions de l'inventeur, sans critique interne ni externe.

La chronologie montre comment les promoteurs en recherche de légitimation de leur initiative et de relais institutionnels se sont alliés précocement: aux fractions ministérielles en souffrance dès mi-1989 (premier séminaire de Mahitsy), notamment les agents du SNVA, en attente d'un renouvellement thématique que la recherche officielle n'apportait pas ; ainsi qu'aux nouveaux décideurs politiques, notamment le ministre de l'Agriculture et les experts de la Banque mondiale et de I'USAID. Friands d'innovations, les bailleurs s'identifiaient aux mêmes valeurs que celles des organisations de la société civile (esprit associatif, autonomie de décision, environnement, esprit compétitif, développement plus participatif et centré sur la communauté locale, lutte contre la pauvreté, etc.). Dans le contexte de crise agricole et de défaut de renouvellement des propositions de la recherche, le SRI, bien que non documenté, apparaissait comme une innovation prometteuse. En pariant sur le SRI, innovation encore sans parrain diplomatique, I'USAID - acteur nouveau venu - pouvait facilement accéder aux arènes locales du développement en périphérie d'aires protégées.

Les structures ministérielles soumises aux injonctions de désengagement et reconfiguration de leurs rôles ont rapidement manifesté un 
intérêt pour un partenariat avec les ONG. Leur rôle était désormais de maintenir l'harmonie entre les divers intervenants, d'officialiser les décisions, et de fournir un cadre officiel, plutôt que d'arbitrer. La Banque mondiale ayant manifesté son enthousiasme en 1995 (BM, 1995), l'État a suivi sans délai, alors que la coopération française restait circonspecte (Vallois, 1996). L'adoption officielle du SRI par le PNVA s'est faite dans le cadre de l'Année nationale du Riz, événement suscité notamment par Laulanié (Tiersonnier, 2001). Un compromis au ministère a permis d'adopter la nouvelle norme vary maroanaka ou SRI pour les rizières « à bonne maîtrise d'eau ", sans abandonner la norme antérieure MAR, réservée aux autres rizières. Celle-ci a été rebaptisée ultérieurement SRA, selon la norme couplée SRI-SRA toujours en vigueur. Aujourd'hui, les projets américains et les ONG appuient surtout le SRI (c'est-à-dire fournissent aux paysans des appuis conditionnés par cette pratique uniquement), quand les projets soutenus au niveau européen appuient conjointement SRI et SRA.

\section{L'internationalisation}

Le SRI a été posé ensuite en doctrine alternative de riziculture pour l'environnement par Norman Uphoff, un universitaire américain renommé, socio-économiste spécialiste des institutions et politiques agricoles, et directeur du CIIFAD. Dans un premier article en anglais (Uphoff, 1999), la rhétorique SRI de Laulanié est conservée presque mot pour mot mais augmentée d'une référence à l'agro-écologie ${ }^{19}$. Le référentiel international agro-écologique disposait d'un "créneau vacant» dans le domaine rizicole. Sans travaux dédiés ni compétences particulières, N. Uphoff mobilise donc l'agro-écologie pour une nouvelle interprétation du " miracle » SRI, en évoquant l'effet de " synergies agro-écologiques ». II insiste désormais sur l'importance du fumier et sur le caractère néfaste de l'engrais minéral, ajoutant aux prescriptions de Laulanié le recours à la fumure organique. II s'engage ensuite dans une campagne d'internationalisation en organisant aussi des recherches d'optimisation à

\footnotetext{
${ }^{19}$ L'article de Razakamiaramanana (1995) n'est jamais cité par Uphoff, et absent du site SRI de Cornell.
} 
Madagascar (université, FOFIFA) puis des colloques SRI dans le monde, et en fondant le réseau "SRI-rice ». Le SRI devient un " cadre de recherches » dans lequel s'engouffrent de multiples disciplines, intéressées par l'optimisation du SRI ou par l'ouverture de " boîtes noires » expliquant le fonctionnement du riz et du sol « sous SRI ».

Ces " champions " internationaux du SRI qu'ont été N. Uphoff en Asie ou W.A. Stoop en Afrique (qui a plus mis l'accent sur l'intérêt du SRI pour les paysans pauvres en ressources, voir Stoop et al., 2002), ont ainsi délivré les éléments de langage pour charpenter le discours de multiples projets. Ces nouvelles qualifications " agro-écologique " et "pro-poor " ont été suivies d'une nouvelle argumentation adaptée aux nouveaux agendas de l'eau (productivité de l'eau) et du climat (réduction d'émission de GES). Elles ont permis de nouvelles concrétisations institutionnelles dans un contexte de plus en plus porteur, à défaut d'adoptions massives de terrain. Les projets promouvant l'agro-écologie à Madagascar n'ont d'autre choix que d'en passer par le SRI.

Cette diffusion mondiale a produit des effets majeurs en retour, et notamment l'élargissement du cercle des soutiens institutionnels et financiers à la sphère privée ${ }^{20}$, la multiplication des projets, ONG porteuses et recherches de toutes sortes dans de multiples pays, ainsi que la relance des efforts de diffusion à Madagascar en tant que pays d'origine (montage d'un groupement " SRI », etc.).

\section{La controverse}

Une controverse éclate en 2004 avec ce processus d'internationalisation vers I'Asie. Opposant des agronomes internationaux proches de I'IRRI (Dobermann, 2004 ; Sheehy et al., 2004 ; Sinclair et Cassman, 2004) aux promoteurs du SRI, cette polémique a eu toutefois très peu d'impact. C'est sur cette base de connaissances contestées, et malgré les constats de désadoption par les paysans, que le CIIFAD puis le réseau "SRI-rice " de I'université Cornell appuient la diffusion mondiale du SRI ainsi que « ses principes » appliqués à d'autres plantes cultivées. Le SRI est désormais

\footnotetext{
${ }^{20}$ Ainsi la fondation Better U de l'acteur Jim Carrey appuie le groupement SRI malgache.
} 
candidat au référentiel " climate-smart agriculture » visant conjointement sécurité alimentaire, résilience et atténuation du changement climatique (Styger et Uphoff, 2016).

\section{Discussion}

Nous n'avons pas rencontré tous les acteurs malgaches actuels du groupement SRI engagé avec le réseau international SRI-rice. Ce biais conforte le point de vue très " extérieur » de notre analyse et en limite de ce fait certainement la portée pour ce qui est d'une analyse sur les processus actuels, qui reste à mener. Néanmoins l'article porte surtout sur la phase malgache antérieure à 1995 et le début de l'internationalisation. Dans ce processus de montée du SRI vers une " norme internationale de développement " se sont associés des mécanismes cognitifs et des facteurs sociopolitiques. Pour en comprendre la nature, discutons nos hypothèses à la lumière de l'étude empirique et du double cadre d'analyse choisi.

\section{Une innovation...}

L'émergence subite du SRI dans les journaux malgaches des années 1991 à 1993 et les annonces ronflantes de ses propriétés prodigieuses laissaient une impression de "légende urbaine ". Mais l'analyse permet de considérer aussi qu'il s'agit également d'une "vraie innovation" et d'abord d'un nouveau savoir-faire.

Le SRI est un itinéraire technique inconnu à Madagascar avant 1987. C'est donc une invention, une alternative. Celle-ci a d'abord mis l'accent sur le repiquage de très jeunes plants à faible densité, puis s'est affinée et précisée jusqu'en 1993, et continue d'évoluer à travers le réseau de recherche mondial SRI-rice. Le SRI et ses variantes élargissent donc indéniablement l'éventail des systèmes de riziculture disponibles. Les cas d'adoption authentique dans les situations les plus favorables (des agriculteurs aisés, des néoruraux, des femmes, des rizières fertiles et bien maîtrisées en eau) suffisent pour considérer le SRI comme une pratique innovante. Comparé aux autres pratiques (locales, SRA), le SRI a montré quelques avantages palpables (moins de semence, moins d'eau en phase 
initiale, rendement accru dans les conditions déjà les plus favorables, toutes autres choses égales, ce qui mène bien à des records de rendement). Dans les situations sans incitations mais aisées (près des routes et marchés), I'intérêt du SRI sur sols fertiles et les changements sociaux en cours (promotion des femmes, néoruraux, marché du travail) produisent des formes d'adoption authentiques, bien que rares. On peut prévoir encore d'autres raisons d'adopter le SRI, si celles-ci devenaient valorisantes. C'est le cas de la filière biologique (Serpantié et Rakotondramanana, 2013), ou d'une moindre consommation d'eau si l'eau était payante. On est donc loin d'une mystification pure.

\section{... devenue mythe}

La dimension mythique du SRI vient de l'occultation active de son autre face à travers un discours de justification peu équitable. Car il n'a pas que des avantages. Longtemps, ses inconvénients et ses coûts ont été ignorés ou niés. Notre propre évaluation confirme l'analyse de Tsujimoto et al. (2009) sur les confusions d'effets " fertilité du sol » et « technique SRI » sur le rendement. Elle complète les conclusions de Razakamiaramanana (1995) sur la faible augmentation de rendement, ainsi que celles de Moser et Barrett (2003) sur les coûts. Le SRI présente des défauts rédhibitoires pour la majorité des agriculteurs de la région d'étude (rendements toutes choses égales inférieurs ou inchangés dans les conditions sous-optimales qui sont les plus fréquentes, et productivité du travail faible toutes choses égales). Ces graves inconvénients suffisent à expliquer le faible intérêt de la majorité des paysans, sauf en cas de mesures incitatives. Finalement I'adoption du SRI encore embryonnaire révèle surtout l'effet des incitations et des avantages sociaux que l'engouement institutionnel permet de financer. Ce type d'adoption est donc précaire et dépendant, et les incitations conditionnées mènent à des inégalités de traitement entre paysans.

Les effets environnementaux de cette adoption sont aussi à réévaluer. Le flux financier des projets repose largement sur la rhétorique de " records de rendement, économie en intrants et en eau, agro-écologie ". Avec les pratiques observées de sur-fertilisation du SRI et même de "verse " parfois constatée (Serpantié et Rakotondramanana, 2013), ces pratiques et actions incitatives contredisent la rhétorique de promotion 
comme la sagesse écologique et économique de répartir la fumure disponible sur les parcelles et ne pas viser des rendements trop élevés. Plus généralement, une politique agricole normative basée sur le lancement à l'échelle nationale de "produits " comme le SRI ou le SRA reste inadaptée aux effets variables de ces itinéraires techniques, qui appellent l'identification de domaines de recommandation pour chacune de ces innovations.

Tant que ce n'est pas fait, le SRI comme le SRA, soumis au seul " plaidoyer " consistant à énumérer leurs prétendus avantages sur le prétendu "système traditionnel ", conserveront une part mythique. Les chercheurs qui auraient pu identifier ces domaines de validité n'ont pas été soutenus, car cela remettait en cause ce type de politique normative, facile à financer. Et les bailleurs de fonds appuient toujours plus les porteurs d'innovations, que leurs critiques.

\section{Les fabricants du mythe}

Si le $S R I$ a réussi à devenir un thème phare jusqu'à un niveau international, malgré ses défauts, c'est qu'il a d'abord convaincu son précurseur et des courtiers. II y a d'abord eu cette fascinante " auto-promotion " par l'apparence du riz et la propriété du SRI de produire des records. L'illusion de l'inventeur est aussi un reflet de sa formation agronomique des années 1930. La "phytotechnie " enseignait le rendement comme un produit direct des techniques, favorisant des généralisations abusives (Sebillotte, 1974). Ici, la création d'un système de culture n'a pas été suivie de son évaluation équitable, la démarche agronomique a donc été tronquée.

Entre les articles successifs de Laulanié, on peut voir sa conviction grandir, sous les effets de la maturation de sa théorie et de son succès politique et médiatique, donc par les effets réflexifs de la diffusion institutionnelle. L'inventeur ne voyait pas l'intérêt de mobiliser les agronomes officiels rivaux, jugés dépassés. Il a cherché à théoriser sa découverte en mobilisant plutôt des sciences spécialisées sur la plante et le sol et en évitant les forums scientifiques. À partir de 1992, la discipline la plus concernée, l'agronomie, qui pouvait être critique, a été politiquement écartée. 
La croyance s'autonomisait après l'adoption politique qui refermait un débat qui n'avait pas eu lieu. Le SRI devenait alors, en 1995, vérité politique. Seul subsistait un régime de plaidoyer pour l'expansion du dogme, qui parvenait à absorber la recherche elle-même, invitée à optimiser le SRI et fournir de nouveaux arguments en accord avec les nouveaux agendas internationaux. Les promoteurs influents, détenteurs d'autorité dans leur institution d'appartenance (prêtres, responsables de centres de recherche, autorités ministérielles), ont réussi, avec énergie et constance, à installer une "orthodoxie de pensée " qui ne fonctionnait qu'en occultant une partie du sujet (ici, les conditions de réussite et les coûts) et en profitant du manque d'information. Les moyens utilisés étaient médiatiques (rhétorique emphatique et imagée, "unes " de journaux locaux, "droits de réponse "), cognitifs (théorisation physiologique puis agro-écologique) et institutionnels (les séminaires de Mahitsy, le lobbying, l'intimidation, la censure).

Le mythe s'est ensuite renforcé, le SRI étant personnifié à Madagascar à travers la figure tutélaire du Père Laulanié, personnalité respectée dont la contribution à l'innovation, la formation et le développement, était par ailleurs reconnue et distinguée par l'État (Tiersonnier, 2001). Cependant, sans $\mathrm{P}$. Vallois puis N. Uphoff qui ont relayé l'inventeur en lui donnant une audience locale dans les ONG puis mondiale, le mouvement serait sans doute retombé faute de partenaires techniques et financiers. La promotion internationale a représenté un travail considérable entre théorisation, médiatisation (sites web, SRI-summits, réseautage, etc.) et mobilisation institutionnelle. On mesure l'énergie énorme dépensée (et les coûts) dans cette entreprise permanente de croissance de la start-up "SRI », financée sur de simples perspectives, et le caractère remarquable des personnalités qui s'y sont investies. Avec l'inflation de pays impliqués dans le réseau SRIrice et de publications, le mythe est devenu global. En se développant à l'international, il s'est réactivé à Madagascar.

\section{Les conditions contextuelles d'émergence du mythe}

La dynamique de promotion et d'adoption politique du modèle SRI résulte d'abord de circonstances favorables à l'adoption de thématiques alternatives de développement: une "fenêtre d'opportunité " liée à une coïncidence entre une offre technique insuffisante et une forte attente de 
renouvellement de la politique agricole dans un contexte de rupture politique et de crise internationale du modèle "Révolution verte ". De même, le nouveau libéralisme appelait des initiatives et médias pluralistes et était propice à l'émergence de "courtiers", de "champions ", voire d' " entrepreneurs", qui ont à la fois encouragé les promoteurs et les demandeurs en vantant le SRI jusqu'à une échelle mondiale.

Ce modèle technique issu de la recherche d'un agronome "non gouvernemental » a ensuite profité de l'ouverture politique pro-ONG des années 1990 à Madagascar et d'un soutien américain. Les organisations de la société civile et leurs appuis internationaux en recherche d'un " segment de marché du développement ", se sont emparés de cette idée originale et l'ont idéalisée pour s'en servir de thème emblématique, et le faire adopter comme norme du développement au niveau national puis international, via le référentiel « agro-écologique».

Ce qui a permis l'adoption officielle, c'est aussi un nouveau régime politique renforçant les fractions religieuses et pro-démocratie de l'élite urbaine qui cherchait à capter la nouvelle aide au développement américaine ou internationale tout en tirant une légitimité d'action au niveau national.

Enfin, le SRI a profité du nouveau paysage scientifique "post-Rio » centré sur l'écologie et les sciences humaines et sociales, partenaires des agendas de la participation et de la biodiversité au Sommet de la Terre. II était désormais facile de marginaliser activement l'agronomie, déjà déstabilisée par la réduction de ses moyens, son caractère non académique (donc, pour certains, non scientifique) et l'inadaptation de ses préconisations anciennes aux nouveaux contextes.

Dans ce processus, l'intérêt de la majorité des paysans et de la production, l'équité dans la distribution des appuis, comme l'efficience des financements du développement, ont été des préoccupations secondaires voire absentes.

\section{Un nouveau "savoir autorisé »}

Le SRI est une illustration des changements constatés dans les politiques du savoir agricole avec l'accent donné aux notions de participation et de 
durabilité (Sumberg et al., 2012). Le principal changement est une multiplication des sources des connaissances considérées comme légitimes. Le savoir autorisé a été diversifié, révélant un processus de démocratisation des sciences (Callon et al., 2001). Le pouvoir exclusif des centres de recherche publique et des universités de produire de la connaissance institutionnalisable a été partagé avec les organisations de la société civile et le secteur privé. À cette fin, l'institution agronomique d'État, affaiblie par les plans d'ajustement structurel, a été contournée voire intimidée, et les méthodes de mobilisation institutionnelle privilégiées. La rhétorique de promotion d'une ONG, bien que non évaluée, ni par elle-même, ni par la recherche, est devenue une vérité officielle. Ses arguments scientifiques, pourtant valables seulement à l'échelle du pied de riz, ont été deux fois reconnus comme connaissance légitime : par les acteurs politico-financiers qui ont opté pour cette invention dans leurs politiques, et par certaines disciplines et institutions scientifiques (experts de la Banque mondiale, chercheurs en sciences humaines et sociales, agroécologie internationale, Académie malgache).

Le discours de justification du SRI apparaît dans I'histoire du savoir agricole à Madagascar comme un nouveau venu. II s'agit d'un savoir dogmatique, qui ne cherche pas à faire la démonstration de son bien-fondé dans son domaine d'application (la parcelle, l'exploitation agricole), et qui en exclut même l'examen critique ou la mise en débat entre experts: on est pour ou contre, dedans ou dehors, c'est une vérité ou un mensonge. Ce mélange de théorie savante, de conviction, de marketing et de valeurs affichées, contraste avec les autres types de savoirs, scientifiques (appuyés sur la méthode et le débat entre spécialistes) ou locaux (appuyés sur la tradition et l'expérience).

La dimension politique du SRI proposée en hypothèse se vérifie peu, sauf en tant que thème phare des nouveaux venus des arènes malgaches du développement (les ONG et l'USAID). Comme ses collègues "officiels", Laulanié était un agronome du développement, conscient des contraintes économiques et de la dépendance alimentaire de Madagascar (Laulanié, 1993). Ce qui était encore combattu, c'étaient les pratiques locales perçues comme archaïques. L'inventeur reprochait aussi à la recherche officielle malgache son inefficacité (Laulanié, 1993). Il s'agissait donc plus d'une forme de rivalité d'action entre institutions agronomiques du dévelop- 
pement que de contestation « éthique » de l'agronomie de la "Révolution verte $"$.

Enfin, on ne peut s'empêcher de voir dans l'origine du SRI (des étudiants avec leur professeur) et les séminaires SRI de Mahitsy, les "forums hydrides » de Callon et al. (2001), puisque le SRI est bien une option innovante, à laquelle le système scientifique et technique officiel n'avait pas pensé. Mais ces rencontres où tous les pouvoirs étaient invités à écouter la nouvelle parole du riz n'étaient pas si démocratiques. Elles ne servaient pas de lieu de débat, mais de chaire magistrale, de tribune, et de chambre de recrutement.

\section{Conclusion}

La légitimation et la mise en politique de ce savoir alternatif à Madagascar ont été $d$ 'abord le fruit de méthodes de mobilisation institutionnelle plus que de recherche et débat de connaissances. Les bases cognitives sont restées faibles sans être absentes (physiologie végétale et science du sol seulement mobilisées) au profit d'une rhétorique de promotion, reléguant ainsi les intérêts de la production, des paysans, voire de l'environnement au second plan.

Les mécanismes politiques, médiatiques et financiers déclenchés par l'émergence d'une croyance partagée parmi de nombreux acteurs ont fourni des moyens pour inciter de nombreux agriculteurs du monde à essayer le SRI, ce qui lui donnait une chance d'exister en pratique. Les résultats de cette expérimentation de masse révèlent une situation d'échec à Madagascar au regard des promesses de l'inventeur et de ses successeurs. Les adoptions opportunistes (incitations conditionnelles, compétitions, promotion sociale, désir de distinction) dominent, les adoptions par intérêt direct existent mais sont plus rares et restent le fait des plus aisés. En tout cas la "légende urbaine " est devenue une pratique observable, donc une innovation agricole, ce qui nous a permis de l'étudier in situ.

Si la genèse du SRI révèle d'abord une diversification du système de gouvernance de l'agriculture, au profit des ONG et des bailleurs de fonds américains travaillant sur la riziculture au tournant des années 1980-1990, 
la "montée en politique " du modèle SRI à l'international traduit en revanche un changement de priorités avec un intérêt marqué pour la "performance environnementale ». Le SRI déjà qualifié d' " agroécologique " est désormais candidat au référentiel "climate smart agriculture " (Styger et Uphoff, 2016). Pourtant les pratiques SRI observées à Fianarantsoa ne nous sont pas apparues si empreintes de sagesse écologique.

Même s'il reste encore un peu de sectarisme de part et d'autre entre "SRI » et "SRA », le temps a émoussé les sensibilités. Le moment est venu d'aborder les " domaines de recommandations » de ces deux options, sans dénigrer les autres pratiques. L'évaluation multidimensionnelle du SRI dans d'autres situations (régions froides des Hautes Terres, régions chaudes de Madagascar, intérêts pour la filière " bio ") n'est pas achevée. Le SRI peut aussi servir à étudier les rizicultures économes en eau, en le comparant à d'autres modèles qui pourraient répondre aux besoins d'adaptation dans les zones soumises à un retard des pluies telles que les Hautes Terres malgaches du Sud.

Mais, mythe ou innovation, reconnaissons au moins derrière la rhétorique SRI un projet de vie, cherchant l'optimum de l'agro-écosystème. On est encore loin de ce but. Mais n'oublions pas, dans cette quête du graal "agro-écologique ", le point de vue de ceux sans lesquels l'agroécosystème n'existerait tout simplement pas, à savoir le paysan et le consommateur.

\section{Bibliographie}

ADLER E., HAAS P.M., 1992, "Conclusion: Epistemic Communities, World Order, and the Creation of a Reflective Research Program ", International Organization, 46(1), 367-390.

AFRICARE, 2010, More Rice for People, More Water for the Planet, Hyderabad (India), Africare, Oxfam America, WWF, ICRISAT.

AGRIDAPE, 2013, "Le système de riziculture intensive ", Agridape, 29(1) : 36 p. 
ANDRIANAIVO B.B., 2003, "Contribution à l'étude de l'amélioration des techniques de riziculture intensive ", thèse université d'Antananarivo, Département Biologie et écologie végétale, Antananarivo, $85 \mathrm{p}$.

ATS, 2007, Voly Vary Maro Anaka Système de riziculture intensive. Antananarivo, Publications SRI-ATS : 35 p.

BANQUE MONDIALE, 1995, "Agricultural Extension Program Support Project Madagascar », Staff appraisal report, Washington (DC), World Bank.

BOCKEL L., 2005, Politiques publiques et pauvreté à Madagascar. La filière riz, moteur de croissance ou facteur de crise ?, Paris, L'Harmattan, 304 p.

CALLON M., LASCOUMES P., BARTHE Y., 2001, Agir dans un monde incertain. Essai sur la démocratie technique, Paris, Seuil.

DOBERMANN A., 2004, "A critical assessment of the system of rice intensification (SRI) », Agricultural Systems, 79 : 261-281.

DUFOURNET R., ROCHE P., 1967, "L'amélioration de la riziculture ", Revue de Madagascar : 1971-1986.

FOFIFA-ASARECA, 2013, Guide for sustainable irrigated rice production, FOFIFA, Antsirabe, $23 \mathrm{p}$.

FORSYTH T., 1996, "Science, myth and knowledge: testing Himalayan Environmental Degradation in northern Thailand ", Geoforum, 27(3) : 375-392.

GANNON F., SANDRON F., 2003, "Convention de solidarité et intérêt collectif dans une communauté rurale malgache", Colloque Conventions et institutions: approfondissements théoriques et contributions au débat politique, organisé par FORUM et Capitalisme(s) et Démocratie(s), Paris, La Défense, 11-13 décembre 2003.

GILLAIN J., 1992, "MAR (méthode améliorée de riziculture sur les Plateaux malgaches) ", Antsirabe, DRDR, multigr.

GSRI, 2012, http://groupementsrimada.org/fr/, consulté en janvier 2012.

INSRE, 1965, "Enquêtes sur le rendement des rizières dans 23 zones rizicoles de Madagascar ", ministère des Finances et du Commerce, 54 p.

LAULANIÉ H. DE, 1991, "Pour une riziculture scientifique construite sur le schéma de tallage de Katayama ", Lakroa, Fianarantsoa, n²724 May 19th: 5-6, $n^{\circ} 2725$ June 2 nd: 5-6, $n^{\circ} 2726$ June 19th: 5-6. 
LAULANIÉ H. DE, 1993, "Le système de riziculture intensive malgache ", Tropicultura, 11(3) : 110-4.

LAULANIÉ H. DE, 1995, "Les fondements scientifiques du système de riziculture intensive ", Bulletin de l'Académie Nationale des Arts, des Lettres et des Sciences, 73(1-2), 1995 (1997) : 211-4.

LAULANIÉ H. DE, 2003, Le riz à Madagascar. Un développement en dialogue avec les paysans, Paris; Antananarivo, Ambozonaty, Éditions Khartala.

LE BOURDIEC F., 1974, Hommes et paysages du riz à Madagascar, FTM, Antananarivo, $648 \mathrm{p}$.

MAAT H. et GLOVER D., 2012, "Alternative configurations of agronomic experimentation ", in Sumberg J., Thompson J. (éds), Contested agronomy. Agricultural research in a changing world : 131-145.

MAE-UPDR, 2002, " Analyse diagnostic de la filière régionale riz dans l'ensemble du secteur vivrier de la province autonome de Fianarantsoa ", Résumé exécutif, ministère de l'Agriculture et de l'Élevage, Antananarivo, $21 \mathrm{p}$.

MILLEVILLE P., 1987, "Recherches sur les pratiques des agriculteurs ", Les Cahiers de la Recherche-Développement, 16 : 3-6.

MORIN E., 1969, La rumeur d'Orléans, Paris, Seuil.

MOSER C.M., BARRETT C.B., 2003, "The disappointing adoption dynamics of a yield-increasing, low external input technology: the case of SRI in Madagascar ", Agricultural Systems, 76 : 1085-1100.

MOUROUX L., 2008, L'aventure IREDEC à Madagascar, Paris, Antananarivo, L'Harmattan-Tsipika, $349 \mathrm{p}$.

RADANIELINA T., CARRIÈRE, S.M., SERPANTIÉ G., 2014, "Origins, Functions, and Persistence of Crop Biodiversity in the Betsileo Highlands, Madagascar ", Economic Botany, (2013)68 : 123-136.

RANDRIAMAMONJY F., 2009, Histoire de Madagascar, 1895-2002, Antananarivo TPFLM, $496 \mathrm{p}$.

RATSIMBARISON V, WILLIAMS D, 1993, Les jardins de riz de Madagascar, Fiche Syfia, $\mathrm{n}^{\circ} 56$, sept. 1993.

RAUNET M. (éd.), 1993, "Bas-fonds et riziculture ", Actes du séminaire d'Antananarivo, December 9-14th 1991, Montpellier, CIRAD.

RAZAKAMIARAMANANA, 1995, "Le SRI : le Riz Miraculeux », Karoka : 10-12. 
Georges Serpantié

RAZAKAMIARAMANANA, GAUDREAU M.M., 2000, «SRI component under two cropping systems in Madagascar ", American Society of Agronomy Annual meeting, November 5-9, 2000, Minneapolis, Minnesota, USA, poster.

ROLLIN D., 1992, " Le système de riziculture intensive (Katayama-Laulanié) est-il révolutionnaire? », CIRAD, multigr.

SEBILLOTTE M. 1974, «Agronomie et agriculture. Essai d'analyse des tâches de l'agronome ", Cahiers ORSTOM, série Biologie, n 24 : 3-25.

SEBILLOTTE M. 2006, "Penser et agir en agronome », in L'Agronomie aujourd'hui, coll. Synthèses, Paris, Quæ : 1-21.

SERPANTIÉ G., 2013, « Genèse malgache d'un modèle agroécologique : le système de riziculture intensive (SRI) », Cahiers Agricultures, $22: 1-8$.

SERPANTIÉ G., TOILLIER A., 2007, « Dynamiques rurales betsileo à l'origine de la déforestation actuelle ", in SERPANTIÉ G., RASOLOFOHARINORO B.M., CARRIÈRE S. (éds), Transitions agraires, dynamiques écologiques et conservation: le "corridor» Ranomafana-Andringitra (Madagascar), Paris, CITE, IRD : 57-68.

SERPANTIÉ G., RAKOTONDRAMANANA M., 2013, "L'intensification de la riziculture malgache, en pratiques ", Cahiers Agricultures, 22 : 401-410.

SHEEHY J.E., PENG S., DOBERMANN A., MITCHELL P.L., FERRER A., YANG J., ZOU Y., ZHONG X., HUANG J., 2004, "Fantastic yields in the systems of rice intensification: fact or fallacy? ", Field Crop Research, 88 : 1-8.

SINCLAIR T.R., CASSMAN K.G., 2004, "Agronomic UFOs », Field Crop Research, 88(1) : 9-10.

STOOP W.A., UPHOFF N., KASSAM A., 2002, "A review of agricultural research issues raised by the system of rice intensification (SRI) from Madagascar: opportunities for improving farming systems for resource-poor farmers ", Agricultural Systems, $71: 249-274$.

STYGER E., UPHOFF N., 2016, The System of Rice Intensification (SRI): Revisiting Agronomy for a Changing Climate, Climate-Smart Agriculture Practice Brief, Copenhagen, Denmark, CGIAR Research Program on Climate Change, Agriculture and Food Security.

SUMBERG J., THOMPSON J., WOODHOUSE P., 2012, "Why agronomy in the developing world has become contentious ", Agriculture and Human Values : 1-13. 
TIERSONNIER J., 2001, Madagascar. Les missionnaires acteurs du développement, Paris, L'harmattan, $218 \mathrm{p}$.

TSUIMOTO Y., HORIE T., RANDRIAMIHARY H., SHIRAIWA T., HOMMA K., 2009, "Soil management: The key factors for higher productivity in the fields utilizing the system of rice intensification (SRI) in the central highland of Madagascar », Agricultural Systems, $100: 61-71$.

UPHOFF N., 1999, « Agroecological implications of the system of rice intensification (SRI) in Madagascar ", Environment, Development and Sustainability, Dordrecht, Springer, 1 : 297-313

VALLOIS P., 1996, Discours de la méthode du riz, Antananarivo, IPNR/CITE.

VERRIER C., 1999, Autodidaxie et autodidactes: I'infini des possibles, Paris, Anthropos, $230 \mathrm{p}$.

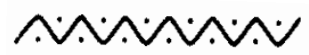

Georges Serpantié est chargé de recherches en agronomie Institut de recherche pour le développement (IRD), UMR GRED

E-mail : georges.serpantie@ird.fr 